is paramount that we consider race and ethnicity in our pursuit to improve antimicrobial stewardship. Racial and ethnic disparities have been well documented in the many healthcare settings, and antibiotic stewardship is no different. ${ }^{9}$ Understanding the role of these factors in different settings will help improve physician training with respect to antibiotic prescribing practices. It can be incorporated into physician implicit-bias training to help mitigate differences in antibiotic prescription practices. It can be utilized to improve community outreach programs and promote patients' understanding of the dangers of antibiotics and how to properly advocate for themselves. As antibiotic resistance becomes a greater threat to our health worldwide, let us not disregard the healthcare disparities that may exist in antimicrobial stewardship. ${ }^{10}$

\section{Acknowledgments.}

Financial support. No financial support was provided relevant to this article.

Conflicts of interest. All authors report no conflicts of interest relevant to this article.

\section{References}

1. Olesen SW, Grad YH. Racial/Ethnic disparities in antimicrobial drug use, United States, 2014-2015. Emer Infect Dis 2018;24:2126-2128.
2. Yang S, Rider BB, Baehr A, Ducoffe AR, Hu DJ. Racial and ethnic disparities in health care-associated Clostridium difficile infections in the United States: state of the science. Am J Infect Control 2016;44:91-96.

3. Flannery DD, Mukhopadhyay S, Jensen EA, et al. Influence of patient characteristics on antibiotic use rates among preterm infants. J Pediatr Infect Dis Soc 2020;44(8):151326.

4. Goyal MK, Johnson TJ, Chamberlain JM, et al. Racial and ethnic differences in antibiotic use for viral illness in emergency departments. Pediatrics 2017;140(2):1-8.

5. Donisi V, Sibani M, Del Piccolo L, et al. Emotional, cognitive and social factors of antimicrobial prescribing: can antimicrobial stewardship intervention be effective without addressing psycho-social factors? J Antimicrob Chemother 2019;74:2844-2847.

6. Fleming-Dutra KE, Hersh AL, Shapiro DJ, et al. Prevalence of inappropriate antibiotic prescriptions among US ambulatory care visits, 2010-2011. JAMA 2016;315:1864-1873.

7. Zoorob R, Grigoryan L, Nash S, Trautner BW. Nonprescription antimicrobial use in a primary care population in the United States. Antimicrob Agents Chemother 2016;60:5527-5532.

8. Grigoryan L, Germanos G, Zoorob R, et al. Use of antibiotics without a prescription in the US population: a scoping review. Ann Intern Med 2019;171:257-263.

9. Gerber JS, Prasad PA, Localio AR, et al. Racial differences in antibiotic prescribing by primary care pediatricians. Pediatrics 2013;131:677-684.

10. Antibiotic resistance. World Health Organization website. https://www. who.int/news-room/fact-sheets/detail/antibiotic-resistance. Published 2020. Accessed October 30, 2020.

\title{
Addressing stigma in the context of the coronavirus disease 2019 (COVID-19) outbreak: A socio-ecological approach
}

\author{
Xuan Qin MSc and Lisheng Song MD \\ Shanghai Mental Health Center, Shanghai Jiaotong University School of Medicine, Shanghai, China
}

To the Editor-The coronavirus disease 2019 (COVID-19) epidemic has sparked social stigma and discrimination against people from certain regions, countries, occupations, or ethnic groups, as well as anyone perceived to have been in contact with the virus. Research on infectious diseases has suggested that stigma presents barriers to prevention, diagnosis, and treatment, increasing physical suffering and psychological burden on the individual who has been victimized in the process. ${ }^{1}$ To describe the stigma that exists and its impact in the context of COVID-19, we provide a taxonomy by employing a socio-ecological model that categorizes the broad lessons learned from communicable diseases into the following levels: intrapersonal, interpersonal, community, institutional, and public policy. ${ }^{2}$ Approaches that address stigma at each level will inform efforts to reduce and control stigma during a pandemic (Fig. 1).

The socio-ecological theory holds that individual factors such as knowledge, attitudes, behaviors, and skills are malleable, constructed with constant feedback from the social environment. ${ }^{3}$ During an epidemic, people develop a relatively consistent set of risk perceptions

Author for correspondence: Dr Lisheng Song, E-mail: slslulu@163.com

Cite this article: Qin X and Song L. (2022). Addressing stigma in the context of the coronavirus disease 2019 (COVID-19) outbreak: A socio-ecological approach. Infection Control \& Hospital Epidemiology, 43: 401-403, https://doi.org/10.1017/ice.2020.1433 through perceived likelihood of infection, personal susceptibility, and disease severity. However, they exhibit individual emotional differences in decision making, especially when confronted with highly uncertain risks. ${ }^{4}$ Existing studies utilizing socio-ecological theory have demonstrated the effectiveness of education as an intervention tool. It is critical that local public-health risk assessments be continuously improved and that they provide real-time, context-sensitive guidance for clinical practice. ${ }^{5}$ Furthermore, psychological assistance is indispensable for all people due to different vulnerabilities and susceptibility to stress. Service providers need to understand the experience and meaning of the disease to the person and to reframe the discourse by empowering the public.

According to social identity theory, the behavioral decisions of potentially stigmatized groups can be influenced not only by personal motivations and skills but also by fear of losing social ties. ${ }^{6}$ COVID-19 has shown the power of continuous human-to-human transmission, so family, friends, neighbors, colleagues, and acquaintances may show euphemistic rejection and ostracism in words and actions, and the expected stigma may make people feel a diminished social identity. ${ }^{7}$ Significant social relationships, under complex changes of the COVID-19 epidemic, need to be constantly adjusted and adapted to bridge differences and enhance

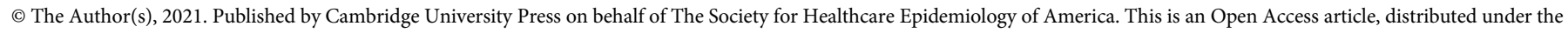

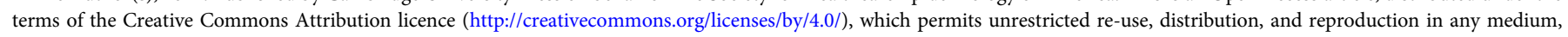
provided the original work is properly cited. 


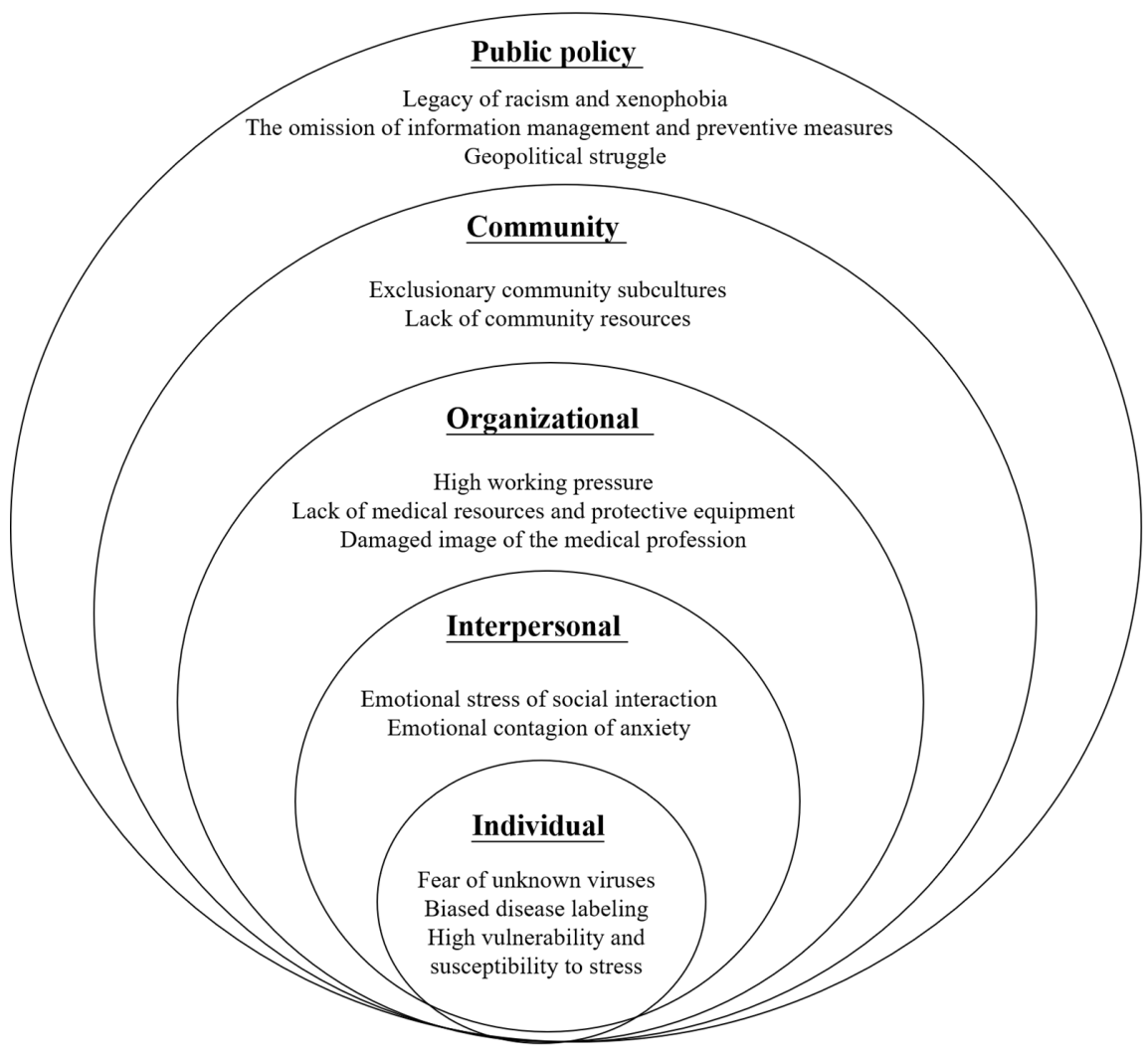

potential interventions

- Implementation of policies based on ethical and humanitarian principles

- Strengthened regulation of information and media

- Protecting and assisting marginalized communities and advocating for global solidarity cooperation

- Involvement of community organizations and members

- Careful, culturally sensitive crisis communication

- Promote inter-community solidarity and a sense of collective effectiveness

- Risk communication and informed consent

- Ongoing educational programs and stress reduction interventions

- Maximizing infection control measures and personal protection training at all levels

- Enhancing organizational cohesion and credibility

- Disease education for families and employers

Maintain communication and provide ongoing emotional support

- Psychological assistance

- Clear guidance on disease education and prevention

- Empowering and encouraging skills development

Fig. 1. The social-ecological taxonomy of infectious disease stigma research.

communication. Support groups, specifically for those who are quarantined, can provide a validating, empowering experience, and a successful interactive experience may encourage people against the anticipated stigmatizing reactions.

In responding to a major public crisis, medical personnel are the most important organizational force, but even well-trained professionals subscribe to stereotypes, especially if they work in high-risk areas. ${ }^{8}$ During the COVID-19 pandemic, HCWs have been heroically at the forefront of the fight against the disease, but many healthcare providers experience considerable stigma and loneliness. Providing direct patient care to infected patients or those who have survived infection may initiate emotional, ethical, and cultural tensions and conflicts for healthcare providers. ${ }^{9}$ It is the responsibility of the organization to create a supportive atmosphere of cohesion and effectiveness and to build a culture of organizational resilience. Importantly, HCWs can be motivated if risks are carefully managed and their professionalism is recognized and affirmed by public figures and the population.

Within a given geographical area, infectious diseases coincide and interact with multiple social concerns, and susceptibility to infectious diseases will expand for members of communities living in poverty with high population density and limited access to health care and other resources. Communities that were once marginalized are at risk of further disenfranchisement. ${ }^{10}$ Given specific networks and subcultures within the community, interventions addressing community stigma need to be adopted in a respectful manner. Community-based organizations and civil society organizations are vital stakeholders, and they should be integrated into a community-based model to eliminate discrimination. In addition, residents may be driven by a greater sense of connectivity and solidarity to engage in collective action to address community issues.

Examining the development of the outbreak narrative, stigma can be regarded as a form of "structural violence," with policies having profound social roots and politics, economic, and ethnocultural contexts. If not applied wisely, the policy burden associated with pandemics can affect the most vulnerable populations, can exacerbate existing inequalities, and can even contribute to more abuse and violence on a global scale. The political exploitation of public policies against global contagion in some countries and regions sparks social comparisons and devaluations of other groups, which make innocent people suffer stagnation because of their nationality, ethnicity or other reasons. ${ }^{11}$ Policy makers and health authorities should be alert to the rise of prejudice and stereotyping of particular societal groups, and they should continually review possible omissions in the way laws, social services, resource allocation, and the justice system are structured for restoring the trust of marginalized communities. Notably, globalization has resulted in international responsibility, and the challenge of COVID-19 requires sustained attention and a strengthened commitment to international equity and inclusion.

Overall, communicable diseases are generally prone to stigmatization, and stigma poses significant challenges to infected individuals, families, social networks, health networks, institutions and communities. In the specific context of a pandemic, stigma cannot contain the virus. Every level is important, and the fight against the COVID-19 pandemic will only be won when the world forms a community of mutual recognition, respect, and reciprocity toward a common destiny. 


\section{Acknowledgments.}

Financial support. This work was supported by National Precision Cooperation Project (grant no. 2017YFC0910000) and by the Clinical Research Project of Shanghai Mental Health Center (grant no. CRC2017YB03).

Conflicts of interest. All authors report no conflicts of interest relevant to this article.

\section{References}

1. O'Neill S, Irani J, Siewe Fodjo JN, et al. Stigma and epilepsy in onchocerciasis-endemic regions in Africa: a review and recommendations from the onchocerciasis-associated epilepsy working group. Infect Dis Poverty 2019;8:34.

2. Stokols D. Translating social ecological theory into guidelines for community health promotion. Am J Health Promot 1996;10:282-298.

3. Rüsch N, Angermeyer MC, Corrigan PW. Mental illness stigma: concepts, consequences, and initiatives to reduce stigma. Eur Psychiatry 2005;20: $529-539$.
4. Adhikari B, Kaehler N, Raut S, Marahatta S, Ggyanwali K. Risk factors of stigma related to leprosy-a systematic review. J Manmohan Mem Inst Health Sci 2014;1:(2):3-11.

5. Heymann DL. Data sharing and outbreaks: best practice exemplified. Lancet 2020;395:469-470.

6. Pryce J, Mableson HE, Choudhary R, et al. Assessing the feasibility of integration of self-care for filarial lymphoedema into existing community leprosy self-help groups in Nepal. BMC Pub Health 2018;18:201.

7. Zhao S, Zhuang Z, Ran J, et al. The association between domestic train transportation and novel coronavirus $(2019-\mathrm{nCoV})$ outbreak in China from 2019 to 2020: a data-driven correlational report. Travel Med Infect Dis 2020;33:101568.

8. Engelbrecht M, Rau A, Kigozi G, et al. Waiting to inhale: factors associated with healthcare workers' fears of occupationally acquired tuberculosis (TB). BMC Infect Dis 2019;28;19:475.

9. Chen Q, Liang M, Li Y, et al. Mental health care for medical staff in China during the COVID-19 outbreak. Lancet Psychiatr 2020;7(4):e15-e16.

10. Stein RA. COVID-19 and rationally layered social distancing. Int J Clin Pract 2020;74(7):e13501.

11. Hirschberger G. Collective trauma and the social construction of meaning. Front Psychol 2018;9:1441.

\title{
The future of masking
}

\author{
Leonard A. Mermel DO, ScM, AM (Hon), FSHEA, FIDSA, FACP ${ }^{1,2,3}$ (1) \\ ${ }^{1}$ Department of Medicine, Warren Alpert Medical School of Brown University, Providence, Rhode Island, ${ }^{2}$ Division of Infectious Diseases, Rhode Island Hospital, \\ Providence, Rhode Island and ${ }^{3}$ Department of Epidemiology and Infection Control, Rhode Island Hospital, Providence, Rhode Island
}

To the Editor-The 2 mRNA vaccines now administered in the United States offer $>90 \%$ protection against symptomatic coronavirus disease 2019 (COVID-19) infection based on published data. How long the protection lasts beyond a few months is uncertain at the present time. There is one report of COVID-19 reinfection in an otherwise healthy adult 6 months after the initial infection despite production of neutralizing antibodies after the first infection. ${ }^{1}$ This individual apparently spread COVID-19 while reinfected, likely reflecting viral replication in the nares in the absence of neutralizing antibodies at that site (ie, lack of mucosal immunity). Widespread vaccination will inevitably reduce the COVID-19 reproductive number, thereby changing the transmission dynamics in many parts of the world. However, infection among those unvaccinated and reinfection in unvaccinated and vaccinated individuals remains an ongoing concern.

Universal masking, along with other public health measures, slows COVID-19 transmission. 2,3 These interventions have also reduced transmission of other respiratory viruses over the last year, ${ }^{4}$ thereby reducing associated morbidity and mortality. ${ }^{5}$ The durability of protection against COVID-19 infection after vaccination is unclear, and universal masking is associated with reduced risk of COVID-19 and infection from other respiratory viruses. What will be the role of masking in the future?

Universal masking should continue until COVID-19 herd immunity is reached from natural disease and vaccination.

Author for correspondence: Dr Leonard Mermel, E-mail: lmermel@lifespan.org Cite this article: Mermel LA. (2022). The future of masking. Infection Control \& Hospital Epidemiology, 43: 403-404, https://doi.org/10.1017/ice.2020.1439
Thereafter, particularly during winter months, ${ }^{6,7}$ universal masking should continue in congregate settings and other indoor settings where social distancing cannot be maintained and/or the introduction of fresh air introduced into the environment, or filtration of recirculated air, is suboptimal. ${ }^{8}$ Mask wearing will be particularly important for those individuals at greatest risk of poor outcomes with COVID-19 infection. ${ }^{9}$ By reducing transmission of respiratory viruses, masking should reduce immune selection pressure in infected individuals, and lower the likelihood that severe acute respiratory coronavirus virus 2 (SARS-CoV-2) will become more transmissible in the future. ${ }^{10}$ There will be temptation to lower our guard and not follow advice about social distancing and masking after vaccination. However, we must learn from our experience over the past year. Failure to maintain more than a modicum of masking would reflect an inability to learn from the past to improve our lives in the future.

\section{Acknowledgments.}

Financial support. No financial support was provided relevant to this article.

Conflicts of interest. All authors report no conflicts of interest relevant to this article.

\section{References}

1. Selhorst P, Van Ierssel S, Michiels J, et al. Symptomatic SARS-CoV-2 reinfection of a healthcare worker in a Belgian nosocomial outbreak despite primary neutralizing antibody response. Clin Infect Dis 2020. doi: 10.1093/cid/ ciaa1850.

2. Leung NHL, Chu DKW, Shiu EYC, et al. Respiratory virus shedding in exhaled breath and efficacy of face masks. Nat Med 2020. doi: 10.1038/ s41591-020-0843-2. 